\title{
Análisis microestructural y mecánico de la ZAC en uniones soldadas CMT robotizadas en acero aleado HSLA
}

\author{
Julio C. García ${ }^{\text {a, }}$, Benjamín Vargas ${ }^{a}$, Celso E. Cruz ${ }^{\text {, }}$ Verónica Estrella ${ }^{\text {a }}$ Irineo P. Zaragoza ${ }^{a}$ \\ ${ }^{a}$ Tecnológico Nacional de México / IT de Tlalnepantla, División de estudios de posgrado e investigación, Av. Instituto Tecnológico s/n, \\ C.P. 54070, Col. La comunidad Tlalnepantla de Baz, Edo. México, México. \\ ${ }^{b}$ Centro de Ingeniería y Desarrollo Industrial (CIDESI) sede Estado de México, Av. Desarrollo s/n Parque Industrial Cuamatla, C.P \\ 54763, Cuautitlán Izcalli, Edo de México, México. \\ *cesarmartinez0816@gmail.com
}

\section{Resumen}

Se estudió el efecto de treinta y uno combinaciones de parámetros de soldadura robotizada por transferencia de metal en frio (CMT-P): corriente de soldadura, velocidad de soldadura, precalentamiento y caudal de gases de protección, sobre la calidad, microestructura y resistencia mecánica bajo tensión en uniones soldadas a traslape con un cordón por cada lado para acero experimental aleado HSLA al Ni-Cr. Se realizaron inspección visual para evaluar calidad de uniones, estereoscopia para análisis macroestructural, microscopia óptica para evolución microestructural y prueba de tensión axial para determinar carga máxima a la tensión. Los resultados indicaron que corrida CMT24 alcanzo la mayor carga máxima a tensión $(27208 \mathrm{~N})$ con probeta de unión soldada fracturada en metal base, así como menor ancho promedio de ZAC $(1.47 \mathrm{~mm})$ en comparación con valor máximo $(2.19 \mathrm{~mm})$ para prueba CMT10 y cordones de soldadura con adecuada calidad sin defectos, como resultado de fases microestructurales duras tales como ferrita acicular en cordón de soldadura y ferrita de grano grueso en la ZAC recristalizada próxima a límite de fusión del cordón. Por lo tanto, esta corrida fue la mejor combinación de parámetros para aplicar soldadura CMT-P robotizada en acero HSLA.

Palabras clave: Proceso CMT robotizado, Acero HSLA, Ancho de ZAC, ferrita acicular.

\section{Introducción}

El acero aleado al Ni-Cr es de alta resistencia baja aleación (HSLA) utilizado en oleoductos, gaseoductos y máquinas agrícolas por alto nivel de tenacidad y resistencia [1]. El efecto del Ni es aumentar la tenacidad y ductilidad, además combinado con $\mathrm{Cr}$ mejora la templabilidad y resistencia al desgaste [2]. Además, aleantes como $\mathrm{Ti}, \mathrm{Cu}, \mathrm{V}$ y $\mathrm{Nb}$ favorecen la soldabilidad, así mismo forman carburos y nitruros proporcionando fortalecimiento en resistencia mecánica final mediante refinamiento de grano y endurecimiento por precipitación, al igual que proceso de laminación en caliente resultando resistencia a la cedencia con variaciones de 290 a $760 \mathrm{MPa}$ y resistencia máxima a la tracción de 410 a 830 MPa [1,3]. Sin embargo, el normalizado, temple y revenido pueden mejorar tenacidad [4].

El proceso de soldadura por transferencia de metal en frio (CMT), incluye corto circuito caracterizado por mantener temperaturas bajas y sin efectos de salpicadura [5], fue desarrollado por empresa Fronius de Austria en 2004 modificando proceso GMAW (MIG) mediante control de parámetros que influyen en deposición del metal y bajo calor de entrada $\left(\mathrm{Q}_{\text {net }}\right)$, resultando sistema digital para control de velocidad de alimentación del alambre de soldadura junto con fase del arco que permiten generar suficiente energía para fundir metales base y de aporte [6]. Una característica del proceso CMT es control digital del alambre con función de avanzar, generando arco eléctrico, que a su vez es retraído apagándose y al mismo tiempo haciendo deposición de gota, generando ciclo alterno (caliente y frio) hasta setenta veces por segundo, dando como resultado uniones de calidad eliminando mecanizados adicionales posteriores [7].

Pickin y Young [8] estudiaron procesos CMT convencional y pulsado (P) para placa de aleación de aluminio AA6061-T6, obteniendo que el convencional transfiere metal de aporte mediante corto circuito, donde penetración disminuye a medida que aumenta número de corto circuitos. Sin embargo, CMT-P proporciona adición de gota por pulsos, donde corriente proporcionada por estos pulsos es mayor y profundidad de penetración depende de aumento de pulsos. Illescas et al [9] estudiaron dos aceros de alta resistencia $16 \mathrm{MnNi} 4(\mathrm{~V}+\mathrm{Nb})$ y $16 \mathrm{Mn} 4(\mathrm{~V})$ evaluando microestructura de acuerdo al $\% \mathrm{~V}$ y propiedades mecánicas de bainita generada, resultando formación de ferrita acicular con valores bajos de dureza y resistencia, así mismo 
mejorando la tenacidad. Pichardo y López [10] evaluaron propiedades microestructurales de junta soldada de acero API-X60 con electrodos de acero inoxidable ER316L para raíz y ER70S-6 como relleno, encontrando fases de perlita y ferrita comunes en aceros HSLA. En unión observaron variación del tamaño de grano en la ZAC por cordones de relleno, encontrando martensita de bajo carbono debido al rápido enfriamiento $\mathrm{y}$ porcentaje de elementos aleantes.

Sin embargo, existe limitada investigación sobre la metalurgia de la soldadura CMT considerando la calidad estas uniones soldadas a traslape de un cordón por lado, caracterización del ancho de ZAC promedio convencional, evolución macro y microestructural de la ZAC y metal de soldadura, y resistencia mecánica bajo tensión axial. El objetivo de esta investigación es el análisis metalúrgico del proceso CMT para identificar la mejor combinación de parámetros de soldadura en uniones soldadas a traslape sobre acero HSLA, así como producir uniones de buena calidad y reducir los defectos.

\section{Parte experimental}

\subsection{Materiales}

Para análisis químico de placa de acero HSLA aleado al Ni-Cr experimental y alambre de soldadura ER70S-6 se utilizó espectrometría de emisión óptica para obtención de composición química, de acuerdo al estándar AWS A5.18 [11], ver tabla 1 incluyendo valor de carbono equivalente $\left(\mathrm{C}_{\mathrm{eq}}\right)$ calculado con ecuación 1 [12] mediante elementos aleantes para determinar soldabilidad del acero y alambre como unión soldada. Analizando este parámetro y $\% \mathrm{C}$ para cada aleación contra diagrama de Graville [13], resulto que acero HSLA con valor de 0.86 se localizó en zona III (difícil soldabilidad), mientras que metal de aporte con 0.31 se ubicó en zona I (fácil soldabilidad), por lo que esta unión tiene limitada soldabilidad. Por lo anterior, se recomienda precalentamiento antes del proceso de soldadura CMT para garantizar adecuada fusión y penetración entre metal de aporte y acero aleado. También, podría aplicarse tratamiento térmico post-soldadura.

Tabla 1. Composición química (\% peso) del metal de aporte AWS ER70S-6 y acero HSLA al Ni-Cr.

\begin{tabular}{|c|c|c|c|c|c|c|c|c|c|}
\hline Material & $\mathbf{C}$ & $\mathbf{M n}$ & $\mathbf{S i}$ & $\mathbf{N i}$ & $\mathbf{C r}$ & $\mathbf{M o}$ & $\mathbf{P}$ & $\mathbf{S}$ & $\mathbf{C}_{\mathbf{e q}}$ \\
\hline $\begin{array}{c}\text { Alambre } \\
\text { ER70S6 }\end{array}$ & 0.10 & 1.15 & 0.41 & 0.15 & 0.15 & 0.150 & 0.002 & 0.008 & 0.31 \\
\hline $\begin{array}{c}\text { Acero } \\
\text { HSLA }\end{array}$ & 0.27 & 1.34 & 0.42 & 1.82 & 0.53 & 0.544 & $\mathbf{0 . 0 1 5}$ & $\mathbf{0 . 0 0 3}$ & $\mathbf{0 . 8 6}$ \\
\hline
\end{tabular}

$C e q=\% C+\frac{\% M n}{6}+\frac{\% N i}{15}+\frac{\% C r}{5}+\frac{\% C u}{13}+\frac{\% M o}{4}$
En tabla 2 se presentan propiedades mecánicas del acero aleado y metal de aporte. Es evidente, que metal base obtuvo mayores valores de dureza HRC y resistencia mecánica bajo tensión debido a elementos aleantes (razón de $\mathrm{Ni}$ a $\mathrm{Cr}$ es 2.5 partes de $\mathrm{Ni}$ por 1 parte de $\mathrm{Cr}$ ) comparándolo con alambre ER70S6. Sin embargo, la ductilidad del metal de aporte fue mayor que la del acero HSLA.

Tabla 2. Propiedades mecánicas del alambre ER70S6 y acero HSLA aleado.

\begin{tabular}{|c|c|c|c|c|}
\hline Material & Dureza & $\begin{array}{c}\mathbf{S}_{\mathbf{0}} \\
(\mathbf{M P a})\end{array}$ & $\begin{array}{c}\mathbf{S}_{\mathbf{U}} \\
(\mathbf{M P a})\end{array}$ & $\begin{array}{c}\text { Elongación } \\
(\%)\end{array}$ \\
\hline $\begin{array}{c}\text { Alambre } \\
\text { ERS70S6 }\end{array}$ & $\begin{array}{c}92.6 \\
\mathrm{HRB}\end{array}$ & 430 & 530 & 21 \\
\hline $\begin{array}{c}\text { Acero al Cr- } \\
\mathrm{Ni}\end{array}$ & $\begin{array}{c}32.3 \\
\mathrm{HRC}\end{array}$ & 777 & 1243 & 14 \\
\hline
\end{tabular}

\subsection{Proceso de soldadura CMT-P robotizado}

Se utilizó placa de acero HSLA al Ni-Cr experimental con dimensiones de $110 \times 110 \times 4 \mathrm{~mm}$ de largo, ancho y espesor, respectivamente, para formar ensambles a traslape (figura 1a). La unión se hizo soldando parte inferior y superior del traslape, aplicando un solo cordón de soldadura por cada lado, ver figura $1 b$ ). Unión de soldadura presento longitud de traslape de $25.4 \mathrm{~mm}$.

Ensambles de placa fueron unidos con proceso CMT-P con arco pulsado $(5 \mathrm{~Hz})$ utilizando como metal de aporte ER70S6 de 1.2 $\mathrm{mm}$ de diámetro, aplicando mezcla de gases: $95 \%$ Ar y $5 \% \mathrm{CO}_{2}, 23 \mathrm{~V}$ y velocidad de alimentación de alambre de $97 \mathrm{~mm} / \mathrm{s}$. Este proceso se realizó mediante brazo robótico Yaskawa 1400 con fuente de alimentación Fronius TransPuls 4000.

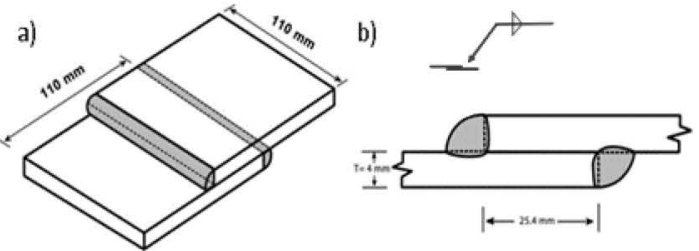

Figura 1. Esquemas de unión soldada CMT-P robotizada en placas de acero HSLA: a) union a traslape y b) union con dos cordones de soldadura.

Se desarrolló diseño de experimentos $\mathrm{DOE}$ aplicando diseño central compuesto mediante software Minitab 18, 2017, considerando cuatro factores con dos niveles (alto y bajo) resultando treinta y uno combinaciones, como se muestra en tabla 3 donde se observa que corriente de soldadura no excede $172 \mathrm{~A}$, lo cual indico bajo aporte de calor de entrada $\left(\mathrm{Q}_{\text {net }}\right)$, velocidad de avance de robot con $77 \mathrm{~mm} / \mathrm{s}$, ya que al 
aumentarla podrían aparecer defectos como faltas de fusión y penetración del cordón de soldadura, caudal de gases menor a $8.5 \mathrm{~L} / \mathrm{min}$ y precalentamiento hasta $250^{\circ} \mathrm{C}$ atendiendo recomendaciones para aceros con baja soldabilidad.

Se diseñó trayectoria lineal para unión de placas programado desde controlador manual, guardando posición inicial y final seguido de instrucción Arcon/Arcof para encendido y apagado de antorcha. Por último, ensambles fueron limpiados con acetona en zona de aplicación del cordón, sujetándolas a mesa de trabajo y posicionando robot para inicio de soldadura CMT-P. En tabla 3, solo se incluyen trece corridas experimentales consideradas como críticas para este análisis.

Tabla 3. DOE central compuesto para pruebas de soldadura CMT-P.

\begin{tabular}{|c|c|c|c|c|}
\hline Condición & $\begin{array}{c}\text { Corriente } \\
\text { de } \\
\text { soldadura } \\
(\mathbf{A})\end{array}$ & $\begin{array}{c}\text { Avance de } \\
\text { soldadura } \\
(\mathbf{m m} / \mathbf{s})\end{array}$ & $\begin{array}{c}\text { Caudal } \\
\text { de gases } \\
(\mathbf{L} / \mathbf{m i n})\end{array}$ & $\begin{array}{c}\text { Pre- } \\
\text { calentamiento } \\
\left({ }^{\circ} \mathbf{C}\right)\end{array}$ \\
\hline CMT-09 & 154 & 63 & 7 & 200 \\
\hline CMT-23 & 163 & 70 & 7.5 & 50 \\
\hline CMT-03 & 154 & 77 & 7 & 100 \\
\hline CMT-01 & 154 & 63 & 7 & 100 \\
\hline CMT-24 & 163 & 70 & 7.5 & 250 \\
\hline CMT-22 & 163 & 70 & 8.5 & 150 \\
\hline CMT-27 & 163 & 70 & 7.5 & 150 \\
\hline CMT-19 & 163 & 56 & 7.5 & 150 \\
\hline CMT-06 & 172 & 63 & 8 & 100 \\
\hline CMT-12 & 172 & 77 & 7 & 200 \\
\hline CMT-10 & 172 & 63 & 7 & 200 \\
\hline CMT-30 & 163 & 70 & 7.5 & 150 \\
\hline CMT-31 & 163 & 70 & 7.5 & 150 \\
\hline
\end{tabular}

\subsection{Prueba de tensión}

Las probetas de tensión se obtuvieron del centro de cada unión soldada CMT, con mecanizado utilizando máquina de corte por chorro de agua Mitsubishi Electric Supreme, con dimensiones de $142 \times 25.4 \times 4 \mathrm{~mm}$ de largo, ancho y espesor, respectivamente de acuerdo al estándar ASTM E8M [14] para determinar resistencia mecánica. Estas pruebas por triplicado para cada condición de soldadura CMT se ensayaron en maquina electromecánica universal de tensión Instron 4482 con celda de carga de $100 \mathrm{kN}$ bajo rapidez de deformación de $15 \mathrm{~mm} / \mathrm{min}$.

\subsection{Análisis macro y microestructural}

Para esta caracterización se realizó técnica de metalografía de acuerdo al estándar ASTM-E3 [15] a partir de probetas de uniones soldadas CMT de acero HSLA al $\mathrm{Ni}-\mathrm{Cr}$ que se cortaron en máquina por chorro de agua con dimensiones de $25 \times 10 \mathrm{~mm}$ de largo y ancho, respectivamente.
Estas muestras se montaron en baquelita para su manipulación y desbaste con papel abrasivo de diferentes grados. Posteriormente, se pulieron con paño agregando $\mathrm{Al}_{2} \mathrm{O}_{3}$ en agua sobre paño húmedo. Finalmente, muestras fueron atacadas químicamente con Picral (4 g ácido pícrico y 100 $\mathrm{ml}$ alcohol metílico) por método de inmersión durante $45 \mathrm{~s}$ para revelar características microestructurales.

Análisis macroestructural de uniones soldadas CMT fue realizado por inspección visual bajo código AWS D1.1 [16] y estereoscopia, para evaluar calidad, sanidad e identificación de defectos de soldadura. Las micrografías obtenidas se procesaron y analizaron mediante software Image J (analizador de imágenes) con calibración para diferentes escalas de magnificación correspondientes con aumento de cada micrografía, tomada mediante estereoscopio Carl Zeiss Stemi 2000-C conectado a cámara de alta resolución para obtención de imágenes utilizando software Axio Visión 4.8.2. Con este procedimiento se midió ancho de ZAC promedio para cada unión soldada CMT a partir de micrografías tomadas a $10 x$.

Para análisis microestructural se utilizaron muestras metalográficas descritas de uniones soldadas CMT para revelar fases microestructurales del metal de soldadura, ZAC y metal base, mediante microscopio óptico Carl Zeiss Axiovert 40 MAT con cámara y software mencionados. Las micrografías de diferentes uniones soldadas se tomaron a 500x.

\section{Resultados y discusiones}

\subsection{Análisis macroestructural de uniones CMT-P robotizadas}

Después de inspeccionar visualmente la calidad en términos de geometría del cordón de soldadura, defectos (falta de fusión y porosidad), ancho promedio y morfología de la ZAC para treinta y uno uniones soldadas a traslape CMT-P con metal de aporte ER70S6, se seleccionaron tres corridas con adecuada calidad (CMT24, CMT27 y CMT22) y otra unión con defecto (CMT6), ver figura 2. Por lo tanto, análisis de resultados se basó en estas cuatro uniones. Adicionalmente, se observó que morfología de la ZAC generada por cordón de soldadura no fue uniforme, resultando dos áreas: semi-elíptica y otra rectangular en cada placa de unión del acero aleado, respectivamente. Ambas áreas influyeron en ancho promedio de la ZAC. 
La unión CMT24 (163 A, $70 \mathrm{~mm} / \mathrm{s}, 7.5$ $\mathrm{L} / \min$ y $250^{\circ} \mathrm{C}$ ) mostro cordón de soldadura convexo, completo y tamaño mediano, obteniendo longitud de pierna horizontal de $4.53 \mathrm{~mm}$, vertical de $3.90 \mathrm{~mm}$ y mediana pierna efectiva (incluyendo convexidad) de $3.97 \mathrm{~mm}$. Por lo anterior, resultaron piernas no proporcionales en longitud, formando ZAC no uniforme con ancho promedio de $1.47 \mathrm{~mm}$, ver figura 2a). La condición CMT27 (163 A, $70 \mathrm{~mm} / \mathrm{s}, 7.5 \mathrm{~L} / \mathrm{min}$ y $150^{\circ} \mathrm{C}$ ) exhibió cordón convexo, pequeño por lo que esta incompleto en ambos extremos, longitud de pierna horizontal de $3.44 \mathrm{~mm}$, vertical de 3.65 $\mathrm{mm}$ y menor pierna efectiva de $3.09 \mathrm{~mm}$. Entonces, piernas mostraron longitud proporcional, resultando ancho promedio de ZAC no uniforme de $1.57 \mathrm{~mm}$ (figura $2 \mathrm{~b}$ ). La corrida CMT22 (163 A, $70 \mathrm{~mm} / \mathrm{s}, 8.5 \mathrm{~L} / \mathrm{min}$ y $\left.150^{\circ} \mathrm{C}\right)$ mostro cordón de soldadura pequeño con ambos extremos incompletos, longitud de pierna horizontal de $4.3 \mathrm{~mm}$, vertical de $3.5 \mathrm{~mm}$ y pierna efectiva mediana de $3.46 \mathrm{~mm}$. Por lo tanto, ancho de ZAC (no uniforme con forma semi-eliptica y rectangular) fue $1.73 \mathrm{~mm}$ (figura 2c). Por lo anterior, en estas tres uniones CMT analizadas se reportó adecuada calidad.

Por último, en soldadura CMT-06 (172 A, 63 $\mathrm{mm} / \mathrm{s}, 8 \mathrm{~L} / \mathrm{min}$ y $100^{\circ} \mathrm{C}$ ) se observó cordón con tamaño grande generando mayor longitud de pierna horizontal $(5.52 \mathrm{~mm})$, vertical de $4 \mathrm{~mm}$ y pierna efectiva más convexidad de $4.4 \mathrm{~mm}$ [16], resultando mayor ancho de ZAC no uniforme de $1.77 \mathrm{~mm}$. Sin embargo, se encontró falta de penetración en raíz de unión, lo cual podría relacionarse con bajo precalentamiento $\left(100^{\circ} \mathrm{C}\right)$, como se observa en figura $2 \mathrm{~d}$ ), por lo que esta soldadura mostro calidad no aceptable.
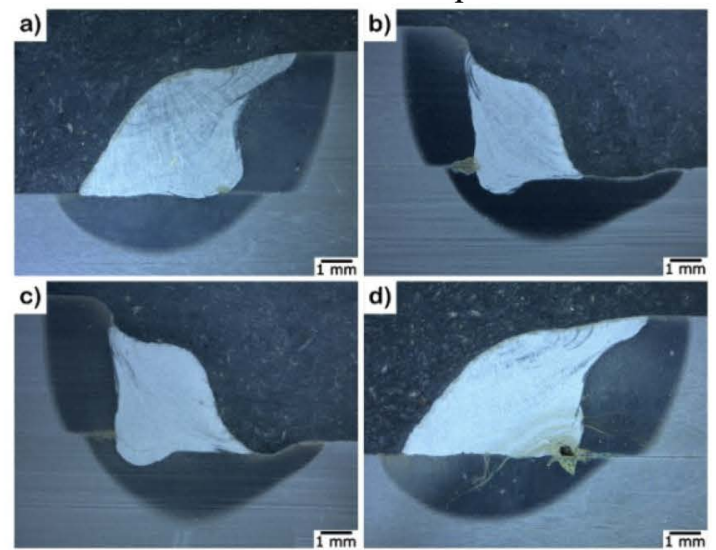

Figura 2 Macrografias obtenidas a 10x para uniones soldadas CMT-P en acero HSLA: a) Cordón mediano con menor ancho de ZAC en CMT24, b) Cordón pequeño con piernas iguales para CMT27, c) Cordón con falta de relleno con piernas desproporcionadas en
CMT 22, y d) Cordón con falta de penetración en raíz y mayor ancho de ZAC en CMT6.

\subsection{Ancho de ZAC en uniones CMT-P robotizadas}

Siguiendo con análisis macroestructural, se midió y analizo ancho promedio de ZAC de treinta y uno corridas CMT-P, resultando nueve condiciones críticas incluyendo valores mínimo, medios y máximo, es decir, rango que va desde 1.34 hasta $2.19 \mathrm{~mm}$, como se observa en figura 3 . Valor mínimo de ancho promedio de ZAC (1.34 $\mathrm{mm})$ se generó con $154 \mathrm{~A}, 77 \mathrm{~mm} / \mathrm{s}, 7 \mathrm{~L} / \mathrm{min}$ y $100^{\circ} \mathrm{C}$ para corrida CMT03, como consecuencia de la menor corriente de soldadura y la mayor velocidad de soldadura. La segunda corrida con menor ancho de ZAC $(1.47 \mathrm{~mm})$ se presentó en GTAW24 atribuido al valor mediano de corriente (163 A), seguida de GTAW 27 con $1.57 \mathrm{~mm}$ debido al precalentamiento mediano $\left(150^{\circ} \mathrm{C}\right)$. Lo anterior, se comparó con el mayor ancho promedio de ZAC $(2.19 \mathrm{~mm})$ derivado de la mayor corriente $(172 \mathrm{~A})$, mayor precalentamiento $\left(200^{\circ} \mathrm{C}\right)$, mediana velocidad de soldadura $(63$ $\mathrm{mm} / \mathrm{s}$ ) y caudal de gases de $7 \mathrm{~L} / \mathrm{min}$, para unión CMT10.

En base a lo anterior, se determinó un aumento en ancho promedio de la ZAC, por lo que el mayor aumento fue encontrado para unión CMT10 (63\%), seguida de CMT27 (17\%) y CMT24 (10\%) en comparación con el menor ancho de ZAC de CMT3, lo cual se atribuyó principalmente con incremento en corriente de soldadura desde 172 , pasando por $163 \mathrm{y}$ hasta 154 A. Por lo tanto, se encontró relación proporcional entre ancho de ZAC y corriente de soldadura.

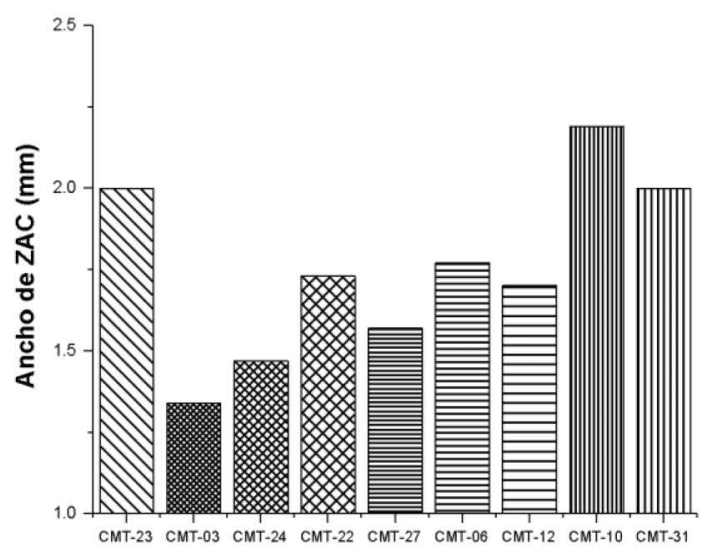

Union soldada CMT

Figura 3. Ancho de ZAC promedio para varias uniones soldadas CMT-P robotizadas en acero HSLA con alambre ER70S6. 


\subsection{Resistencia mecánica bajo tensión de uniones CMT robotizadas}

Después de analizar los resultados de resistencia mecánica medida como carga máxima a la tensión de pruebas de tensión por triplicado para treinta y uno uniones soldadas CMT-P, se determinó rango de carga máxima desde $9558 \mathrm{~N}$ (CMT6) hasta $27280 \mathrm{~N}$ para CMT24. Se identificaron cuatro condiciones representativas: tres corridas (CMT24, CMT22 y CMT27) próximas a la carga máxima de tensión y otra (CMT6) con la menor carga. El comportamiento de tracción representado como curva carga desplazamiento para cuatro uniones CMT indicadas se puede observar en figura 4 , se observa incremento en valores de carga de tensión conforme aumenta desplazamiento para cuatro corridas, de acuerdo con combinaciones de parámetros de soldadura aplicadas. También, es evidente deformación elástica, seguida de deformación plástica con pendiente positiva prolongada, llegando a punto máximo de carga para después tener fractura drástica y repentina, en menor o mayor grado para cada corrida. Además, este comportamiento mecánico bajo tensión podría relacionarse con tipo de unión soldada a traslape con dos cordones.

La mayor curva en términos de carga se encontró para unión CMT24 con la mayor carga máxima a tracción $(27208 \mathrm{~N})$ en forma de pico, seguida de caída drástica para llegar a carga de fractura con menor desplazamiento $(4.85 \mathrm{~mm})$, ver figura 4 , como consecuencia de combinación de parámetros de soldadura CMT-P (corriente mediana de $163 \mathrm{~A}$, velocidad de soldadura intermedia de $70 \mathrm{~mm} / \mathrm{s}, 7.5 \mathrm{~L} / \mathrm{min}$ y alto precalentamiento de $250^{\circ} \mathrm{C}$ ), resultando menor ancho de ZAC $(1.47 \mathrm{~mm})$ y unión con dos cordones convexos sin defectos. Entonces esta unión fue la mejor en términos de ancho de ZAC, adecuada calidad y mayor carga máxima a tensión. La tercer unión con alta carga máxima $(23436 \mathrm{~N})$ se observó en soldadura CMT22 con mayor deformación elástica y plástica, ambas reflejadas como el mayor desplazamiento (6.15 $\mathrm{mm})$ y carga máxima a la tensión con menor pendiente, en comparación con CMT24. La cuarta unión con alta carga máxima $(21275 \mathrm{~N})$ se alcanzó para CMT27 con menores valores de deformación plástica y desplazamiento $(3.05 \mathrm{~mm})$.

Todo lo anterior, se comparó contra curva de unión CMT6 con la menor carga máxima a tensión $(9558 \mathrm{~N})$, así como mínima deformación elástica y plástica resultando el menor desplazamiento $(0.55 \mathrm{~mm})$, ver figura 4 , lo cual se relacionó con alta corriente de $172 \mathrm{~A}$, avance mediano de soldadura de $63 \mathrm{~mm} / \mathrm{s}$, bajo precalentamiento de $100^{\circ} \mathrm{C}$ y $8 \mathrm{~L} / \mathrm{min}$, resultando mayor ancho de ZAC $(1.77 \mathrm{~mm})$ y cordón de soldadura con defecto de falta de penetración. Por lo tanto, esta condición fue la peor soldadura CMT.

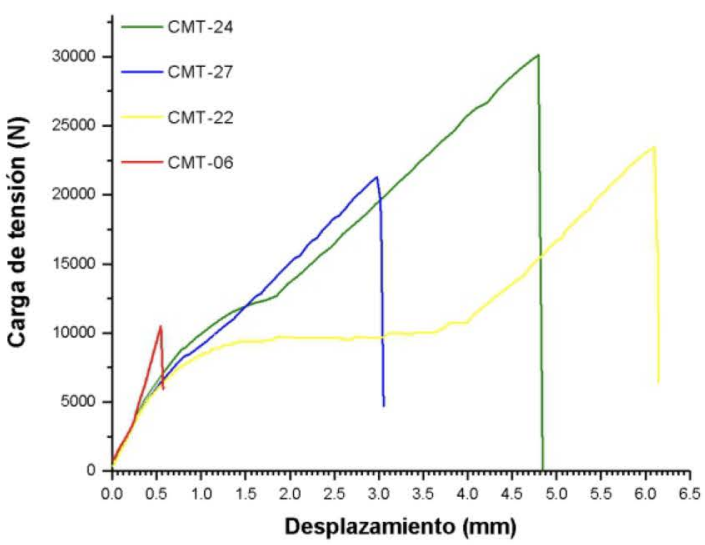

Figura 4. Curvas carga - desplazamiento para cuatro corridas de soldadura robotizada CMT-P.

Siguiendo con análisis mecánico, se analizaron más a detalle nueve condiciones CMT$\mathrm{P}$ en función de carga máxima a la tensión, como se muestra en figura 5. Se confirma rango de esta carga (9558 a 27208 N). Además, se encontró que corrida CMT10 alcanzo carga de $25611 \mathrm{~N}$ y el mayor ancho de ZAC $(2.19 \mathrm{~mm})$ por lo que esta combinación de parámetros CMT no se recomiendan para soldar aceros HSLA. Adicionalmente, soldaduras CMT19 y CMT30 con similar carga máxima $(21785 \mathrm{~N})$ no son adecuadas debido a que la zona de fractura en probetas de tensión fue en la ZAC cuando se requiere que fractura sea en metal base [17], por lo que no se recomiendan ambas combinaciones de parámetros CMT. Entonces, uniones CMT14, CMT22 y CMT27 siguen siendo condiciones en segundo, tercer y cuarto lugar por debajo de CMT24. Sin embargo, carga máxima a tensión promedio del acero HSLA original fue $32697 \mathrm{~N}$, por lo que la mayor carga de tensión de mejor unión CMT24 fue menor en $17 \%$ que carga de este acero. 


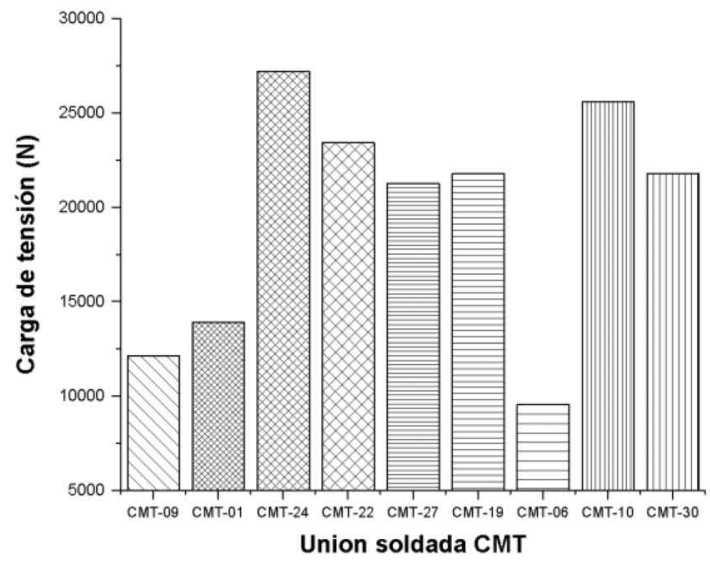

Figura 5. Carga máxima a la tensión promedio para varias uniones soldadas CMT-P robotizadas en acero HSLA.

\subsection{Análisis microestructural de uniones soldadas CMT-P robotizadas}

El análisis del comportamiento mecánico de carga máxima bajo tensión, ancho de ZAC y calidad de uniones soldadas CMT-P, se complementó con evolución microestructural para corridas CMT24 y CMT6. Las características microestructurales de ambas zonas (ZAC y cordón de soldadura) variaron de acuerdo con combinación de parámetros de soldadura robotizada en cada corrida.

Para unión soldada CMT24 transversal (figura 6a) se observó que cordón de soldadura generado con alambre ER70S6 mostro microestructura formada por granos finos de ferrita columnar (zonas blancas) paralelos, intercalados y rodeados de agujas finas de ferrita acicular en mayor cantidad (zonas obscuras) [18], como consecuencia de solidificación del metal de soldadura, ver figura $6 \mathrm{~b}$ ). Mientras que la ZAC fue producto de recristalización cerca del límite de fusión del cordón (ZAC de segunda zona de recristalización), esta compuesta por ferrita de grano grueso y agujas de ferrita acicular [18] distribuidas en matriz ferritica, como se observa en figura 6c). Además, la ZAC cerca del metal base (ZAC de primera zona recristalizada) mostro granos ferriticos muy finos [18] (figura 6d). Estas características microestructurales favorecieron la mayor resistencia mecánica medida como carga máxima a la tensión, menor ancho de ZAC y unión sin defectos, es decir, calidad aceptable, en comparación con treinta y uno corridas CMT analizadas. Por lo anterior, la unión con mejor comportamiento microestructural y mecánico fue CMT24 como resultado de mejor selección de parámetros de soldadura CMT-P.
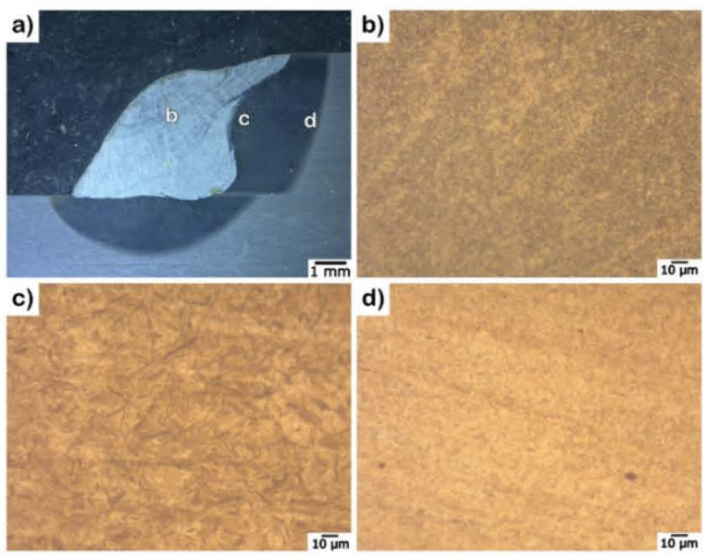

Figura 6. Macrografía por estereoscopia y micrografías obtenidas por microscopía óptica de junta soldada CMT24: a) dos zonas microestructurales en unión, b) ferrita acicular en cordón, c) ferrita acicular en ZAC de $2^{\mathrm{a}}$ zona recristalizada y d) ferrita refinada en ZAC de $1^{\text {ra }}$ zona recristalizada.

En corrida CMT6 transversal (figura 7a) se encontró cordón de soldadura con menor cantidad de ferrita acicular entre granos columnares [18] en mayor proporción, ver figura $7 \mathrm{~b}$ ). $\mathrm{La} \mathrm{ZAC}$ de $2^{\mathrm{a}}$ zona recristalizada mostro baja presencia de ferrita acicular en matriz de ferrita de grano grueso (figura 7c) [18]. Mientras que la ZAC de $1^{\text {ra }}$ zona recristalizada exhibió matriz ferritica con grano fino, ver figura 7d). Este comportamiento microestructural de reducción en fases duras se reflejó en la menor carga máxima a tensión, así como mayor ancho de ZAC y defecto de falta de penetración en cordón de soldadura. Por lo tanto, esta corrida fue la peor de todas las uniones CMTP.
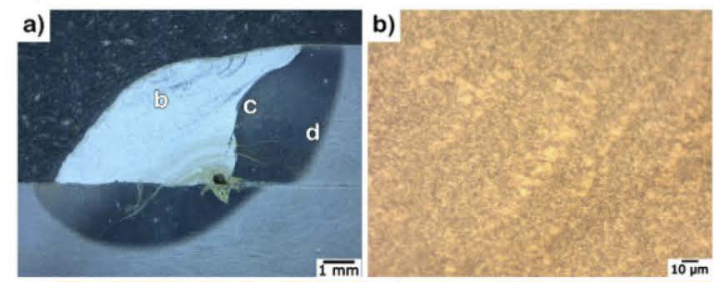

c) d)

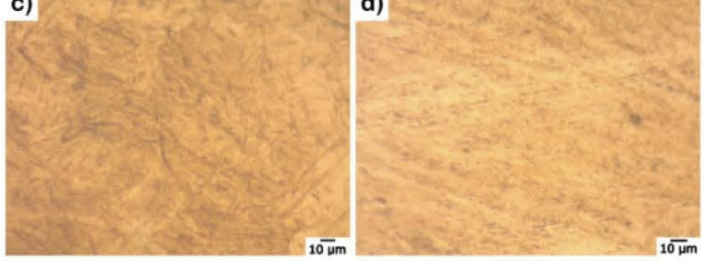

Figura 7. Macrografía por estereoscopia y micrografías obtenidas por microscopía óptica de corrida CMT6: a) cordón con falta de penetración, b) ferrita acicular en cordón, c) ferrita acicular en ZAC de $2^{\mathrm{a}}$ zona recristalizada y d) matriz ferritica refinada en ZAC de $1^{\text {ra }}$ zona recristalizada. 


\section{Conclusiones}

Las uniones a traslape soldadas mediante robot (CMT24, CMT22 y CMT27) mostraron calidad aceptable en términos de cordones de soldadura convexos, valores de ancho promedio de ZAC dentro del rango medido desde $1.34 \mathrm{~mm}$ (CMT3) hasta $2.19 \mathrm{~mm}$ (CMT10), intervalo de carga máxima a la tensión calculado de 9558 N (CMT6) a $27208 \quad \mathrm{~N} \quad$ (CMT24) y características microestructurales de la ZAC recristalizada y cordón de soldadura solidificado.

Por lo tanto, la corrida CMT24 alcanzo menor ancho de ZAC $(1.47 \mathrm{~mm})$ y la mayor carga máxima a la tensión, lo cual se asoció con fases microestructurales duras tales como mayor cantidad de agujas finas de ferrita acicular en cordón y ferrita de grano grueso en la ZAC recristalizada cerca al límite de fusión del metal de soldadura. Todo lo anterior, se atribuyó a la mejor combinación de parámetros de soldadura CMT-P: corriente mediana de soldadura (163 A), avance intermedio de soldadura $(70 \mathrm{~mm} / \mathrm{s})$ y mayor precalentamiento $\left(250^{\circ} \mathrm{C}\right)$. Por lo tanto, esta combinación de parámetros se recomienda para aplicar proceso CMT-P en acero aleado HSLA al $\mathrm{Ni}-\mathrm{Cr}$.

Por otro lado, la peor corrida CMT6 presento la menor carga máxima a tensión, así como mayor ancho de ZAC $(1.77 \mathrm{~mm})$, derivado de menor presencia de ferrita acicular en cordón de soldadura y ZAC de segunda zona recristalizada. Este comportamiento mecánico y microestructural se debió a limitada combinación de parámetros de soldadura: mayor corriente de soldadura (172 A) y menor precalentamiento $\left(100^{\circ} \mathrm{C}\right)$.

\section{Referencias}

1. M. Saadati, On the hot cracking of HSLA steel welds: Role of epitaxial growth and HAZ grain size, Journal of Manufacturing Processes, 2019, pp. 242251
2. S.H. Avner, Introducción a la metalurgia física, New York City, McGraw-Hill, 1988, p. 357.

3. Y. Liu, Effect of step quenching on microstructures and mechanical properties of HSLA steel, Materials Science \& Engineering A, 2016, pp. 371-378

4. AWS Welding Handbook, Carbon and low-alloy steel, 2011, pp. 1-16.

5. H.T Zhang, The arc characteristics and metal transfer behaviour of cold metal transfer and its use in joining aluminium to zinc-coated steel, Materials Science and Engineering A, 2007, pp. 111-113.

6. S. Selvi, Cold metal transfer (CMT) Technology an overview, Defence Technology, 2018, pp. 28-44.

7. J. Arevalo, Proceso de soldadura por transferencia de metal en frio, Research Gate, 2008, pp. 1-5.

8. C.G. Pickin and K. Young, Evaluation of cold metal transfer (CMT) process for welding aluminium alloy, Science and technology of welding and joining, 2006. vol. $11, \mathrm{n}^{\circ} 5$, pp. 583585.

9. S. Illescas, J. Fernández, J. Asensio, M. Sánchez S. J.M. Guilemany, Study of the mechanical properties of low carbon content HSLA, Revista de Metalurgia, 2009, pp. 01-08.

10. B.A. Pichardo A., V.H. López M., R. García H., M. Salazar M., Microestructura y propiedades mecanicas de una junta soldada de placas bicapas API X60/316L, Ava Cient., 2019, vol. VII, No. 2, pp. 135-145.

11. AWS A5.18, Specification for carbon steel electrodes and rods for gas shielded arc welding, American Welding Society, Miami Florida USA 2005, pp. 138-146.

12. AWS A1.1, Metric practice guide for the welding industry, American Welding Society, Miami Florida USA, 1998, pp. 1-16.

13. B.R. Somers, Handbook Welding brazing and soldering, ASM, 1993, pp. 1064.

14. ASTM E8/E8M, Standard test methods for tension testing of metallic materials, ASTM USA, 2016.

15. ASTM E3, Standard guide for preparation of metallographics specimens, ASTM USA, 2011.

16. AWS D1.1, Structural welding code, American Welding Society USA, 2015.

17. ASME, Boiler and pressure vessel code, ASME BPVC IX, 2015.

18. G. Thewlis, Classification and quantification of microestructures in steels, Materials Science and Tecnology, 2004, 20, pp. 143-159. 